\title{
Tumor carcinoide de apéndice con cuadro clínico de apendicitis aguda, intervenido de urgencia por apendicetomía por laparoscopia. Presentación de caso clínico
}

\author{
Carcinoid tumor of the appendix with clinical picture of acute appendicitis, urgently \\ operated on by laparoscopic appendectomy. Presentation of clinical case
}

Donovan Reynoso-Saldaña*, Ricardo Reynoso-González, Gregorio J. Jiménez-Gamas y

Jesús G. Valencia-Martínez

Servicio de Cirugía General, Hospital Regional Tlalnepantla, Instituto de Seguridad Social del Estado de México y Municipios, Tlalnepantla de Baz, Estado de México, México

\begin{abstract}
Resumen
Los tumores de apéndice cecal son extremadamente raros (menos del 0.5\%), y los tumores carcinoides se describen como el tipo de tumor apendicular más frecuente. Se reporta el caso de un paciente de 32 años, sin antecedentes patológicos de importancia, con datos de abdomen agudo. Se decidió apendicectomía laparoscópica, presentando hallazgos de proceso inflamatorio apendicular, con reporte de histopatología de tumor neuroendocrino. Pese a que las neoplasias de apéndice son raras, la apendicitis es una presentación común, y de ahí la importancia de realizar estudios histopatológicos de toda pieza de apéndice cecal obtenida.
\end{abstract}

Palabras clave: Abdomen agudo. Apendicitis aguda. Tumor carcinoide. Laparoscopia.

\begin{abstract}
Cecal appendix tumors are extremely rare (less than 0.5\%), with carcinoid tumors being described as the most common type of appendix tumor. A case of a 32-year-old male patient is reported, with no significant pathological history, with acute abdomen data. Laparoscopic appendectomy was decided, presenting findings of an appendicular inflammatory process, resulting in a neuroendocrine tumor histopathology report. Despite the fact that appendix neoplasias are rare, appendicitis is a common presentation, hence the importance of conducting histopathology studies on every piece of cecal appendix obtained.
\end{abstract}

Keywords: Acute abdomen. Acute appendicitis. Carcinoid tumor. Laparoscopy.

\footnotetext{
Correspondencia:

*Donovan Reynoso-Saldaña

Avda. Paseo del Ferrocarril, 88

Los Reyes Ixtacala, Hab. Los Reyes Ixtacala

Barrio de los Árboles/Barrio de los Héroes

Fecha de recepción: 22-08-2020

Cir Cir. 2022;90(1):120-123

C.P. 54055, Tlalnepantla de Baz, Edo. de Mex., México

Fecha de aceptación: 16-12-2020

Contents available at PubMed

E-mail: reynosodon@ hotmail.com

DOI: $10.24875 / C I R U .200009261$

www.cirugiaycirujanos.com

0009-7411/@ 2020 Academia Mexicana de Cirugía. Publicado por Permanyer. Este es un artículo open access bajo la licencia CC BY-NC-ND (http://creativecommons.org/licenses/by-nc-nd/4.0/).
} 


\section{Introducción}

Los tumores de apéndice cecal son extremadamente raros y menos del $0.5 \%$ de los especímenes de apendicetomía corresponden a cáncer apendicular. Este tipo de tumores rara vez se diagnostican antes de la cirugía y suelen ser hallazgos intraoperatorios o por estudio patológico'. Estadísticamente, la mayoría de los estudios mencionan que se identificaron tumores carcinoides como el tipo de tumor apendicular más frecuente, mientras que el $10-20 \%$ corresponden a adenocarcinoma, adenocarcinoide o linfosarcoma, entre otros ${ }^{2}$.

El tumor carcinoide del apéndice fue descrito por primera vez en 1882 . Su incidencia varía del $0.08 \%$ al $0.1 \%$ de todas las apendicectomías y del $4 \%$ al $6 \%$ de las neoplasias apendiculares malignas. Epidemiológicamente, la edad media de presentación es alrededor de los 50 años, y con predominio en los hombres $^{3}$. La apendicitis es una presentación común en hombres y mujeres, pero la diseminación peritoneal de la neoplasia mucinosa apendicular es una importante presentación inicial ${ }^{4}$. Sin embargo, la hemicolectomía derecha es en la mayoría de las ocasiones el tratamiento de elección. El pronóstico de la neoplasia apendicular está dado por el grado de extensión en la pared apendicular, la existencia o no de ganglios metastásicos y la presencia de metástasis a distancia, sobre todo hepáticas ${ }^{5}$.

\section{Caso clínico}

Varón de 32 años, sin antecedentes patológicos de importancia, que inicia su padecimiento 32 horas previo a su ingreso, con dolor abdominal de tipo cólico en la región del mesogastrio y periumbilical, con escala de dolor 10 de 10. Acude a servicio facultativo y es diagnosticado como infección de vías urinarias, indicando tratamiento médico a base de sulfamidas y antiinflamatorio no esteroideo. Sin embargo, no presenta evolución favorable, motivo por el cual acude al servicio de urgencias de la unidad médica del Hospital Regional Tlalnepantla.

A su ingreso presenta los siguientes signos vitales: frecuencia cardiaca 72 I.p.m., frecuencia respiratoria 16 r.p.m., temperatura $37.5{ }^{\circ} \mathrm{C}$ y presión arterial 103/70 mmHg.

Despierto, consciente, orientado, con discreta deshidratación de mucosas, adecuada coloración de tegumentos, normocéfalo, cuello sin alteraciones, precordio

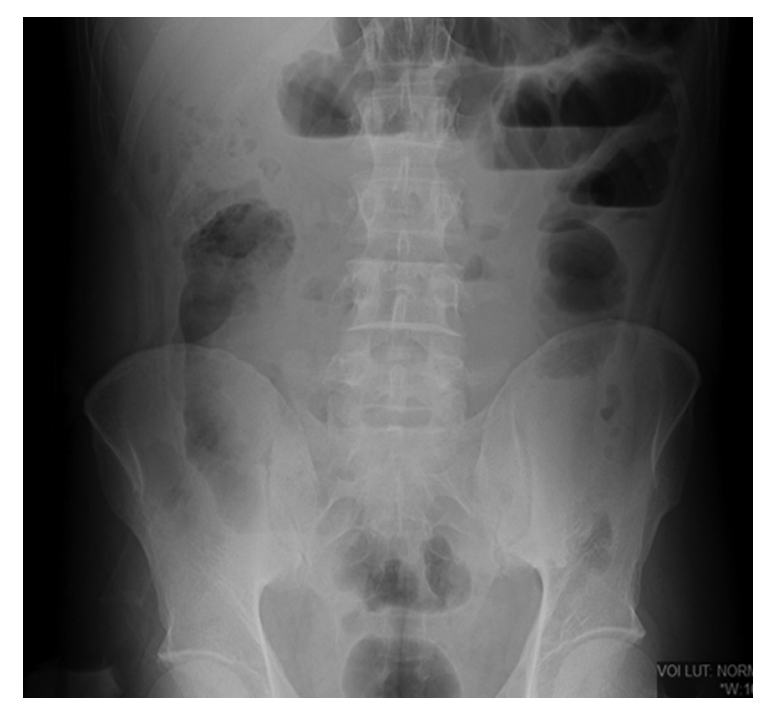

Figura 1. Radiografía de abdomen de pie en la que se aprecian múltiples niveles hidroaéreos, borramiento de psoas bilateral, así como de grasa preperitoneal.

con ruidos cardiacos rítmicos de buena intensidad sin alteraciones, campos pulmonares bien ventilados, sin fenómenos agregados, abdomen plano, sin peristalsis, blando y depresible, doloroso a la palpación en la fosa iliaca derecha, con resistencia muscular voluntaria, McBurney positivo, psoas positivo, obturador y Rovsing positivo, genitales acorde a edad y sexo, extremidades eutróficas, reflejos osteotendinosos presentes, llenado capilar inmediato y pulso distal presente.

La analítica muestra: leucocitos 17.74 , neutrófilos $82.6 \%$, hemoglobina $17.7 \mathrm{~g} / \mathrm{dl}$, hematocrito 53.4 , plaquetas 287.000 , glucosa $106.7 \mathrm{mg} / \mathrm{dl}$, tiempo de protombina $13.4 \mathrm{~s}$, INR 1.24 , tiempo de tromboplastina 29.5. El examen general de orina reporta: $\mathrm{pH} 7$, sedimento con leucos 4-6 por campo, células epiteliales escasas y bacterias escasas.

En la radiografía de abdomen de pie se aprecian múltiples niveles hidroaéreos y borramiento del psoas bilateral, así como de grasa preperitoneal (Fig. 1).

Se decide su hospitalización en el servicio de urgencias a cargo de cirugía general y se inicia manejo con analgésico, cefalosporina de tercera generación y soluciones intravenosas. Se prepara para quirófano y pasa a sala de procedimiento.

Cirugía realizada: apendicectomía laparoscópica. Abordaje con técnica de Hasson a través de una incisión umbilical de $1 \mathrm{~cm}$, posteriormente se coloca un trocar umbilical de $12 \mathrm{~mm}$ y se inicia capnoperitoneo hasta $12 \mathrm{mmHg}$. Se introduce una lente de $30^{\circ}$ y se realiza laparoscopia diagnostica. Se procede a la 


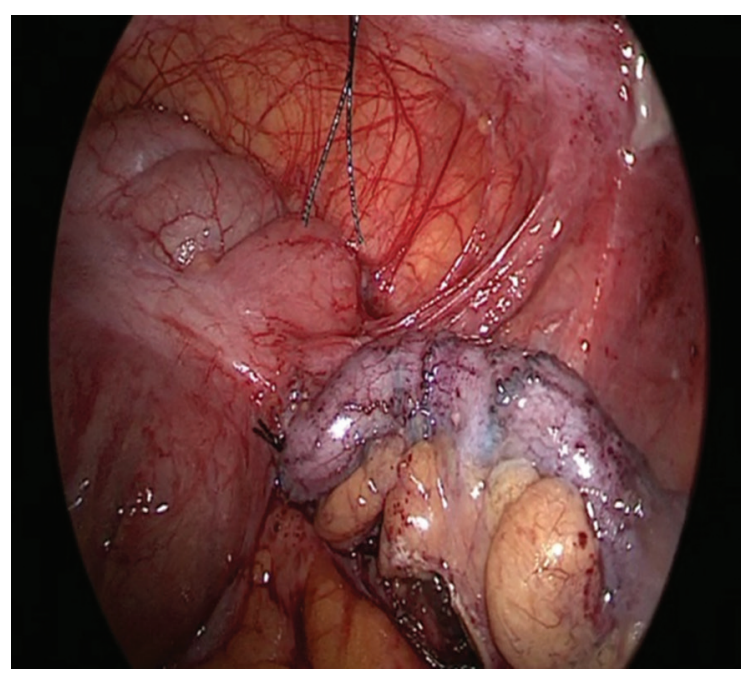

Figura 2. Disección y ligadura de la base del apéndice.

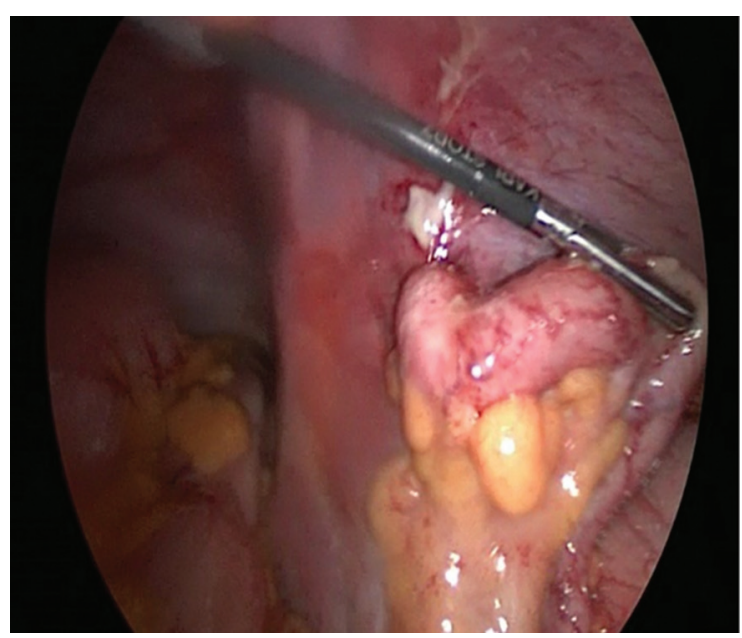

Figura 3. Apendicectomía laparoscópica con majeo de muñón con técnica de Halsted.

colocación de trocares de $5 \mathrm{~mm}$, de región suprapúbica y de flanco izquierdo.

Hallazgos: adherencias laxas del epiplón a la pared. Líquido libre purulento de aproximadamente $20 \mathrm{ml}$. Apéndice vermiforme de $10 \mathrm{~cm}$, hiperémico, edematoso en toda su extensión, con placas fibrinopurulentas. Se realiza apendicectomía y se maneja el muñón con técnica de Halsted. Sin complicaciones, con sangrado mínimo (Figs. 2 and 3 ).

Posterior al evento quirúrgico sin complicaciones, el paciente cursa asintomático, tolerando la vía oral a las 12 horas, canalizando gases y evacuando a las 18 horas. Se decide su egreso con seguimiento ambulatorio.

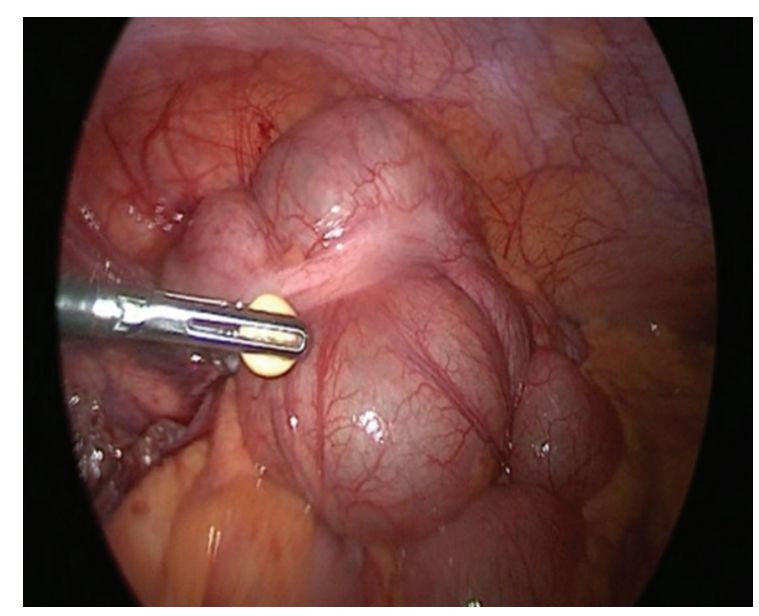

Figura 4. Tumor neuroendocrino (tumor carcinoide apendicular).

La pieza patológica es enviada a estudio en forma ordinaria, recibiendo el resultado con carácter urgente con la siguiente descripción del tejido: mucosa intestinal con epitelio intestinal conservado, lamina propia con hiperplasia, el tejido linfoide con folículos linfoides activos, la serosa congestiva. El tercio medio muestra epitelio colónico conservado y criptas con producción de moco, serosa engrosada con leucostasis, y agregados neutrófilos y mesoapéndice congestivo. Los cortes de la punta muestran neoplasia dispuesta en nidos de aspecto organoide, de células de tamaño mediano, con citoplasma escaso y moderado, nucleos «en sal y pimienta». La extensión del tumor es hasta de $5 \mathrm{~mm}$ en su eje mayor. Discurre a través de la submucosa y a las capas musculares en forma individual, que a los cortes seriados no rebasa la muscular externa, serosa ni mesoapéndice. Tumor neuroendocrino (tumor carcinoide apendicular) (Fig. 4).

\section{Discusión}

El tumor carcinoide es el tipo histológico maligno más frecuente en la patología apendicular, con una prevalencia aproximada del $0.32 \%$ entre los tumores digestivos. Puesto que solo a un mínimo porcentaje de la población se le extrae el apéndice, puede asumirse que la mayoría de los pacientes con carcinoide apendicular no tienen manifestaciones clínicas y no requieren tratamiento, sin que ello afecte su esperanza de vida, a pesar del tumor abdominal ${ }^{5}$.

Aproximadamente el $10 \%$ se ubican en el tercio distal del apéndice cecal, donde tienen poca probabilidad de causar obstrucción; si se localiza en la base del apéndice cecal, puede generar obstrucción y provocar apendicitis. Por este motivo, la mayoría de los 
pacientes permanecen asintomáticos. Los síntomas son más probables en los pacientes con tumores grandes y en casos raros con metástasis más allá de los ganglios linfáticos regionales ${ }^{5}$.

El comportamiento clínico y la evolución dependen, sobre todo, del tamaño del tumor. Los tumores menores de $2 \mathrm{~cm}$ (encontrados en aproximadamente el $95 \%$ de los pacientes) tienen poca probabilidad de metástasis 5 .

Para los carcinoides apendiculares de 1-2 cm, la resección formal del colon derecho no parece mejorar la supervivencia, e incluso en los tumores de grado superior; sin embargo, se sugiere dar seguimiento a estos pacientes si se identifican factores de mal pronóstico, como metástasis ganglionar, invasión linfovascular, invasión mesoapendicular o patología mixta ${ }^{6}$.

Los tumores mayores de $2 \mathrm{~cm}$ producen metástasis a distancia hasta en una tercera parte de los pacientes en el momento del diagnóstico, o en la intervención quirúrgica, que afectan principalmente a los ganglios regionales. Estos tumores también pueden generar invasión al mesoapéndice; en estos, es necesario realizar una hemicolectomía resecando los ganglios linfáticos del apéndice cecal y cualquier enfermedad residual que pudiera quedar en la base del apéndice cecal o en el mesoapéndice. Raramente se producen metástasis hepáticas, pero en los que las presenten debe completarse la hemicolectomía derecha como tratamiento del tumor ${ }^{6}$.

Aunque hay variación en las estrategias y la frecuencia de la vigilancia posoperatoria, Murray et al. ${ }^{7}$ demostraron que no hubo casos de recidiva tumoral ni mortalidad específica de la enfermedad en el periodo de seguimiento, y concluyen que la vigilancia posterior a la resección puede no ser necesaria para tumores $\leq 1 \mathrm{~cm}$ con resección R0.

En la mayoría de las ocasiones, como en nuestro caso, los tumores son tratados eficazmente con apendicectomía y se ha descrito la ausencia de recurrencias y de muertes específicas de la enfermedad en pacientes con una mediana de seguimiento de 5 años, e incluso de 25 años?

\section{Conclusiones}

Los tumores de apéndice cecal son sumamente raros y menos del $0.5 \%$ de los especímenes de apendicetomía se diagnostican como cáncer apendicular. A pesar de que las neoplasias de apéndice son raras, la apendicitis es una presentación común en hombres y mujeres, y de ahí la importancia de realizar estudios histopatológicos de toda pieza de apéndice cecal obtenida. La hemicolectomía derecha es el tratamiento de elección en la mayoría de las ocasiones.

\section{Agradecimientos}

Los autores agradecemos al servicio de cirugía general del Hospital Regional Tlalnepantla, del Instituto de Seguridad Social del Estado de México y Municipios, por el apoyo brindado.

\section{Conflicto de intereses}

Los autores declaramos no tener ningún tipo de conflicto de intereses.

\section{Financiamiento}

Los autores no recibieron patrocinio para llevar a cabo este artículo.

\section{Responsabilidades éticas}

Protección de personas y animales. Los autores declaran que para esta investigación no se han realizado experimentos en seres humanos ni en animales.

Confidencialidad de los datos. Los autores declaran que han seguido los protocolos de su centro de trabajo sobre la publicación de datos de pacientes.

Derecho a la privacidad y consentimiento informado. Los autores han obtenido el consentimiento informado de los pacientes y/o sujetos referidos en el artículo. Este documento obra en poder del autor de correspondencia.

\section{Bibliografía}

1. Das R, Cantor JP, Vu TQ. Two concurrent appendiceal neoplasms in an elderly patient: a case report. Int J Surg Case Rep. 2017;40:124-6.

2. Umetsu SE, Shafizadeh N, Kakar S. Grading and staging mucinous neoplasms of the appendix: a case series and review of the literature. Hum Pathol. 2017;69:81-9.

3. Wu H, Chintagumpala M, Hicks J, Nuchtern JG, Okcu MF, Venkatramani R. Neuroendocrine tumor of the appendix in children. J Pediatr Hematol Oncol. 2017;39:97-102.

4. Nutu OA, Marcacuzco Quinto AA, Manrique Municio A, Justo Alonso I, Calvo Pulido J, García-Conde M, et al. Mucinous appendiceal neoplasms: incidence, diagnosis and surgical treatment. Cir Esp. 2017;95:321-7.

5. Suárez-Grau JM, García-Ruiz S, Rubio-Chaves C, Bustos-Jiménez M, Docobo-Durántez F, Padillo-Ruiz FJ. Appendiceal carcinoid tumors. Evaluation of long-term outcomes in a tertiary level. Cir Cir. 2014;82:142-9.

6. Nussbaum DP, Speicher PJ, Gulack BC, Keenan JE, Ganapathi AM, Englum BR, et al. Management of $1-$ to $2-\mathrm{cm}$ carcinoid tumors of the appendix: using the National Cancer Data Base to address controversies in general surgery. J Am Coll Surg. 2015;220:894-903.

7. Murray SE, Lloyd RV, Sippel RS, Chen H, Oltmann SC. Postoperative surveillance of small appendiceal carcinoid tumors. Am J Surg. 2014;207:342-5 